\title{
What are the greatest challenges and/or barriers to applying postmortem imaging in paediatric radiology?
}

\author{
Stephen Chapman
}

Received: 28 March 2014 / Accepted: 23 April 2014 / Published online: 18 May 2014

(C) Springer-Verlag Berlin Heidelberg 2014

Without accurate final diagnoses we are unable to audit our work and society cannot properly manage its health care systems. Modern imaging is not yet $100 \%$ accurate and in some situations there may be insufficient time to fully investigate. Yet, non-forensic autopsies have become virtually extinct. Postmortem imaging, MRI in particular, with or without minimally invasive autopsy, provides a solution that is more socially acceptable. However, there are a great number of obstacles outside of the well-equipped, well-staffed forensic institute, i.e. where most of us work in the real world. First, access to the scanner for the corpse, both within days of death and at a suitable time of day that does not cause distress to waiting patients. This is difficult when waiting lists for the living are long and scanners already run extended hours. Second, appropriate training of what will initially be a small group of subspecialists. Postmortem imaging is not just the simple transfer of clinical knowledge to postmortem diagnosis. Third, the development of multidisciplinary teams. Failure to do so will result in failure of the entire objective and may risk the specialty becoming part of pathology, not radiology. How many radiologists undertake echocardiography? Finally, money. Hospitals struggling with their budgets will have to be convinced of the benefits when asked to fund such things as increased staff costs, training costs and data storage.

Conflicts of interest None.

S. Chapman $(\bowtie)$

Radiology Department, Birmingham Children's Hospital,

Steelhouse Lane, Birmingham B4 6NH, UK

e-mail: steve.chapman@bch.nhs.uk 